\title{
Documentation of survivors of gender-based violence (GBV)
}

Iffat Idris

GSDRC, University of Birmingham

16 July 2021

\section{Question}

What is the evidence on appropriate methods (both in terms of inclusion and ethics) for recording personal data and feedback of survivors of gender-based violence? Include, if available, use of online counselling and psychosocial services such as helplines.

\section{Contents}
1. Summary
2. Risks in GBV documentation
3. GBV documentation principles
4. GBV Information Management System (GBVIMS)
5. Documentation with remote GBV services
6. References

The K4D helpdesk service provides brief summaries of current research, evidence, and lessons learned. Helpdesk reports are not rigorous or systematic reviews; they are intended to provide an introduction to the most important evidence related to a research question. They draw on a rapid deskbased review of published literature and consultation with subject specialists. other Government departments, but the views and opinions expressed do not necessarily reflect those of FCDO, the UK Government, K4D or any other contributing organisation. For further information, please contact helpdesk@k4d.info. 


\section{Summary}

Documentation of gender-based violence (GBV) cases is important in allowing survivors to access support services and secure justice, and - on a wider scale - for analysis and improvement in GBV responses. GBV documentation carries significant risks for survivors and others, and hence must be taken with care. Key principles include: do no harm, take a survivorcentred approach, ensure non-discrimination and confidentiality, and protect data. The GBV Information Management System (GBVIMS) provides a standardised tool for collecting, analysing and sharing GBV data, and is being implemented in a growing number of developing countries in humanitarian settings. Remote service delivery (phone-based case management, hotlines) pose additional challenges, and require tailored measures to ensure confidentiality and data protection.

This review is largely based on grey literature, in particular policy documents and reports by international development organizations. While there was substantial literature on approaches and principles to GBV documentation, there was less on remote service delivery such as helplines - much of this only in the wake of the COVID-19 pandemic. In addition, very little was found on actual examples of GBV documentation in developing contexts. By definition, gender featured strongly in the available literature; the particular needs of persons with disabilities were also addressed in discussions of overall GBV responses, but far less in GBV documentation.

Key findings of the review are as follows:

- GBV documentation refers to the recording of data on individual GBV incidents in order to provide/refer survivors with/to appropriate support, and the collection of data of GBV incidents for analysis and to improve GBV responses. There are two main sources of quantitative data: administrative sources, and population-based prevalence surveys. ${ }^{1}$ This review only looks at the former.

- There are significant risks associated with GBV documentation, in relation to data protection. Failure to ensure information security can expose survivors, in particular, to harm, e.g. reprisal attacks by perpetrators, stigma and ostracism by their families/ communities. This means that GBV documentation must be carried out with great care.

- A number of principles should always be applied when documenting GBV cases in order to protect survivors and prevent potential negative effects:

- Do no harm - being aware of the risks involved, and putting in place measures to prevent/minimize harm.

- Survivor-centred approach - putting the survivor at the centre of each step of the response process, and making decisions based on the survivor's needs, wishes and capacities.

- Survivor autonomy - enabling and respecting the survivor's right to make his/her own decisions

'(1) Administrative data, including, but not limited to records kept by health and social services, hotlines, shelters, legal aid services, courts and police; and (2) population-based surveys, a term that is used for surveys that collect data through rigorous methods in a subset of the population - a representative sample - to find out what is happening in the entire population.' kNOwVAWdata, 'Sources of Data'.

https://asiapacific.unfpa.org/sites/default/files/pub-pdf/vaw-sources-of-data-28august2020-final.pdf 
- Informed consent - ensuring that a survivor agrees to participate on the basis of: their having full information, including of risks and benefits; them being competent to decide; and no coercion, threats or promises of benefits being used to secure that consent.

- Non-discrimination - GBV survivors receive equal and fair treatment regardless of their age, gender, race, religion, nationality, ethnicity, sexual orientation or any other characteristic.

- Confidentiality - not disclosing any information at any time to any party without the informed consent of the GBV survivor. Confidentiality promotes safety, trust and empowerment. Survivors should also be informed of any exceptions to confidentiality.

- Data protection (information security) - putting in place measures to keep data secure, e.g. never sharing survivor's personal details, anonymising data, using passwords for information stored electronically.

- The GBV Information Management System (GBVIMS) is a data management system that enables those providing services to GBV survivors to effectively and safely collect, store, analyse and share data related to the reported incidents of GBV. It comprises four tools: GBV Classification Tool (which has six core types of GBV); Intake and Consent Forms; Incident Recorder; and Information Sharing Protocol Template. GBVIMS is being used by international and local agencies in a growing number of developing countries. The Primero/GBVIMS+ is a more recent software system which has technological enhancements including heightened security, role-based access and viewing rights.

- Remote GBV service delivery operates over a technology platform (i.e. hotline, chat, or SMS) rather than in person. Two main methods are phone-based case management and by calling hotlines. The COVID-19 pandemic has led to increased use of such remote service delivery, with often both survivors and practitioners at home. These carry additional ethical and security risks which can lead to breaches of confidentiality and privacy, and expose survivors to harm, e.g. others listening in to phone calls, or accessing SMS messages.

- Recommendations to ensure security in remote GBV service delivery include: using only electronic data and not keeping hard copies of GBV case files in practitioners' homes; no call back policy on hotlines; establishing codes/red flag phrases; having different access rights to data for staff, depending on need; following data protection protocols; and including undertakings on data protection in staff contracts.

\section{Risks in GBV documentation}

This review focuses on gender-based violence ${ }^{2}$. GBV is a broad term which includes physical, sexual and psychological abuse of women and girls (and also men and boys) in the home, community, workplace and in schools; trafficking; traditional practices such as female genital

\footnotetext{
2 Defined as violence directed against a person because of that person's gender or violence that affects persons of a particular gender disproportionately. https://ec.europa.eu/info/policies/justice-and-fundamental-rights/genderequality/gender-based-violence/what-gender-based-violence_en
} 
mutilation and 'honour' crimes; and sexual violence and exploitation during and after conflicts and natural disasters (CARE, 2014: 1).

GBV documentation refers to the collection of data of individual GBV incidents (including action plans and follow up actions) by a case management organization (GBV SC Turkey Hub, 2018: 51). Documentation of GBV cases is important in order to enhance quality of services to survivors. However, GBV cases are by their very nature - in both developed and developing countries - highly sensitive. There are real risks involved in reporting and documenting GBV incidents, stemming from shortcomings in data protection and confidentiality. These include:

- Threats and/or reprisal attacks by the perpetrators/their families and friends on survivors/their family and friends/GBV practitioners (e.g. NGO staff)

- Names, addresses and other personal details of survivors being in the public domain (e.g. through the news media, social media)

- Survivors and/or their families being abused/blamed for what has happened to them

- Survivors facing stigma and ostracism in the communities in which they live, possibly leading to loss of employment/education opportunities/other negative consequences

- Survivors facing rejection or even further violence (e.g. so-called 'honour killings') by their families, especially in highly conservative societies where notions of honour are strongly tied to females.

The stigma and marginalization faced by survivors in their own communities can be especially damaging, as elaborated here (OHCHR, 2019: 19):

It may prevent them from accessing critical services, with risks for victims' physical and mental health. It may lead to domestic violence, as well as spousal, family and community abandonment or rejection (including possible separation from one's own children), including retaliation, 'honour killings' or forced and early marriage (including with the perpetrator). Stigmatization and abandonment may lead to loss of resources and livelihoods. Stigma can turn the blame on the victims of sexual violence, who in some cases can be criminalized (e.g. for adultery, same-sex relations). Children born as a result of rape often suffer stigmatization and its consequences.

Indeed, survivors have 'reported that the experience of stigma can be as damaging as the incident of sexual violence itself' (OHCHR, 2019: 19). Stigma is a major barrier and deterrent to the reporting of sexual violence, preventing victims from seeking justice - often very difficult anyway - and accessing support (OHCHR, 2019: 19). A survey on rape in America found that, 'victims are extremely concerned about people finding out and finding reasons to blame them for the rape', which puts them off reporting it (Zannoni, n.d.: 5).

Figure 1 gives an overview of the various ways in which survivors of GBV could face harm. Given these, GBV documentation has to be undertaken with great care: ensuring confidentiality for survivors and protecting data are especially critical. 
Figure 1: Causes of harm for GBV survivors

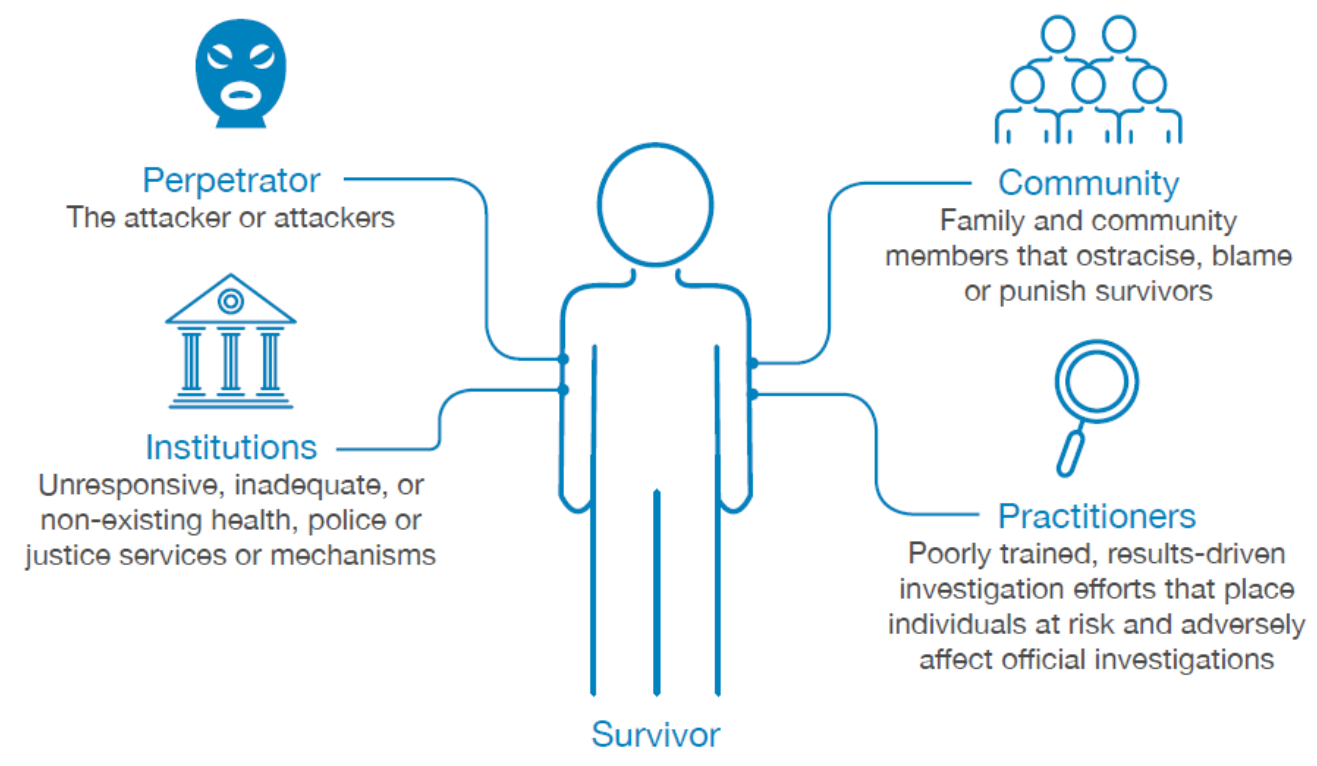

Source: Ribeiro \& Ponthoz, 2017: 86. Licensed under Open Government Licence v3.0

\section{GBV documentation principles}

\section{Overview}

The following key principles, based on a WHO-UNODC 3 toolkit (2014: 11) on medico-legal responses to sexual violence, are applicable to documentation of all forms of GBV:

- The physical safety and emotional well-being of the victim should be the primary consideration.

- The confidentiality of the victim and her/his information must be guaranteed. Where there are legal limitations to confidentiality, this information must be shared with the victim as early as possible, to allow her/him to make an informed decision about disclosure.

- The victim's wishes and rights must be respected. The victim must be informed of her/his options and be able to exercise her/his autonomy to make informed choices (informed consent).

- Individuals, agencies and organizations must not discriminate against victims on any basis, including religion, race, sex, gender identity, age, ethnic group, profession, socioeconomic level, political affiliation or sexual orientation.

- Those interacting with victims must be careful not to make promises and misrepresentations (particularly regarding security) that cannot be guaranteed.

- There must be policies in place to protect those who may not be in a position to legally consent to medical and medico-legal processes.

These principles are explored in more depth below.

\footnotetext{
${ }^{3}$ World Health Organization, and UN Office on Drugs and Crime.
} 


\section{Do no harm}

The key ethical principle at the heart of GBV documentation is the obligation to, at a minimum, 'Do No Harm'. 'This means practitioners must be fully aware of the possible negative impacts of documentation on victims and other witnesses, the wider community and the investigators themselves; be prepared for the harm those impacts may inflict; and put in place measures to prevent or minimize that harm' (Ribeiro \& Ponthoz, 2017: 85).

Ribeiro and Ponthoz (2017: 85) acknowledge that some risks are unavoidable, and investigators cannot offer a complete guarantee of protection and well-being to victims, witnesses or entire communities. However, they stress that the safety and dignity of survivors must be at the centre of the entire documentation process. 'This means ensuring the process prioritises their needs and requests, especially with respect to security concerns' (Ribeiro \& Ponthoz, 2017: 85). Recommended measures for practitioners to protect survivors/witnesses include (Ribeiro \& Ponthoz, 2017: 116):

- Only question victims of sexual violence if strictly necessary and the information is not otherwise available, and limit the number of interviews.

- Inform victims and witnesses about possible risks and security measures which must be adopted, ideally prior to meeting in person.

- Protect the identity of victims/witnesses and conduct interviews in a location that is safe, private, neutral, comfortable, easily accessible and culturally appropriate

- As a general rule, do not record the image or voice of a witness if there are security concerns.

- Do not discuss work/sensitive issues in public, in taxis or in front of drivers, with friends/family members or with colleagues outside of the documentation team.

- Always discuss with the victim/witness the safest way to get in touch and interact as it may put them at risk to call at home, leave messages, send emails or be seen with them.

- Advise victims/witnesses on non-disclosure about the documentation exercise (who can they tell and how much).

- Do not tell victims/witnesses anything about the documentation exercise they do not need to know. Never show victims/witnesses investigative notes, statements or other documents that are unrelated to their evidence.

- Never give witnesses information about other witnesses.

- Be supportive, sensitive and caring in all interactions with victims and witnesses.

The Syria Justice and Accountability Centre (SJAC, n.d.) describes its approach when dealing with survivors/witnesses of sexual and gender-based violence (SGBV):

If collecting documentation would put the interviewee at risk of excessive harm, or if the interviewee has extreme difficulty telling his/her account of the events, then SJAC's documenters do not continue. Additionally, SJAC makes efforts to avoid re-traumatising the interviewee by conducting only one interview and demonstrating sensitivity during the process. 
The latter point is important: survivors should not be further traumatised during the documentation process. The GBV Sub-Cluster in Iraq (n.d.: 13-14) lists the following measures for practitioners dealing with GBV cases in its standard operating procedures:

- Believe the survivor and do not blame her/him for the violence she/he experienced

- Be patient; do not press for more information if the survivor is not ready to speak about her/his experience

- Ask only relevant questions (for example, the status of the virginity of the survivor is not relevant and should not be discussed)

- The same case worker/manager should handle the survivor meetings through her/his case management process.

- Use simple language that the survivor understands.

In its guidelines for documenting GBV cases for communications purposes (e.g. a documentary), CARE International (2014: 2) list similar measures:

- showing sensitivity and respect when seeking out and approaching interviewees;

- interviewing survivors in a safe location (away from family or onlookers, where discussions cannot be overheard);

- if possible, offering the choice of a male or female interviewer;

- if possible, choosing a translator of the same sex.

A further useful measure would be to make provisions for people with special needs, e.g. sign language interpreters for deaf people.

\section{Survivor-centred approach and autonomy}

Taking a survivor-centred approach and ensuring survivor autonomy are strongly linked to the 'do no harm' principle. A survivor-centred approach is defined as follows (GBV Sub-Cluster Turkey Hub-Syria, 2018: 24):

the survivor should be placed at the centre of each step of the response process, and that every decision should be driven by the survivor's needs, wishes and capacities. The survivor-centred approach aims to create a supportive environment in which a survivor's rights are respected and in which the survivor is treated with dignity and respect. This approach helps promote a survivor's recovery and empower them to make decisions about possible recovery interventions.

Ribeiro and Ponthoz (2017: 88) define survivor autonomy as 'a survivor's ability to make her or his own decisions, and respect for autonomy requires the recognition of a person's right to make independent choices, and take action based on their own personal values and beliefs'. This could result in survivors making decisions which the practitioners regard as harmful and self-injurious, but even then survivor autonomy must be respected.

\section{Informed consent}

Informed consent means that a person agrees to participate in an activity, or for something to occur after s/he has knowledge of, or has received, all the information about the activity (GBV SC 
Iraq, n.d.: 19). Informed consent is a legal and ethical obligation of anyone gathering information about crimes under international law. Obtaining informed consent before documenting testimonial evidence ensures that the survivor/witness maintains full control and power over her/his own experiences, and that $\mathrm{s} / \mathrm{he}$ is a knowledgeable and willing participant in the justice process. Conversely, failure to do so violates the rights of the survivor/witness, disrespecting her/him and causing her/him harm (Ribeiro \& Ponthoz, 2017: 90).

In addition, sharing information with survivors about risks and benefits of participation in, for example, legal proceedings, as well as what these entail, and making them aware of/referring them to support services, can help them to make optimal decisions.

In order to give informed consent, a person must:

- Have all the information

- Be over the legal age required to give consent

- Be mentally sound enough to understand the agreement and the consequences

- Have equal power in the relationship.

With regard to the third condition listed above, the ability to give voluntary informed consent is based on a general requirement of competency. Some people may not be competent to provide informed consent (Ribeiro \& Ponthoz, 2017: 89):

- Adult men and women with severe intellectual disabilities, mental illness, or any other physical, mental or emotional condition that may impair their ability to fully comprehend all the relevant facts.

- Children are generally presumed to lack competency to consent, but depending on their age and other factors, such as maturity, may be required to provide informed assent.

Where a survivor is incapable of giving informed consent, the permission of a legally authorized representative must be sought in lieu.

To ensure consent is informed, service providers must explain (GBV SC Iraq, n.d.: 20):

- All the options that are available

- That information (as agreed with the survivor) will be shared with others in order to access other services; $s$ /he has the right to place limitations on the type(s) of information to be shared, and to specify which organizations can and cannot be given the information.

- Exactly what is going to happen as a result of accepting other services

- The benefits and risks of the service

- That survivors have a right to decline or refuse any part of the service offered

- Mandatory reporting policies

- Information in such a way that persons with disabilities understand it, using alternate means of communication where necessary.

There is no consent when agreement is obtained through (GBV SC Iraq, n.d.: 20):

- The use of threats, force or other forms of coercion, abduction, fraud, manipulation, deception, or misrepresentation 
- The use of a threat to withhold a benefit to which the person is already entitled to

- A promise is made to the person to provide a benefit.

Informed consent is a process and involves consenting throughout all stages of documentation, which may include any of the following (Ribeiro \& Ponthoz, 2017: 90):

- to be interviewed and/or examined

- to be photographed

- to have their information recorded

- to be referred to any support services

- to have their information and contact details shared with third parties.

Informed consent must never be assumed, and must be obtained in writing.

\section{Non-discrimination}

GBV survivors have the right to non-discrimination. This means they 'should receive equal and fair treatment regardless of their age, gender, race, religion, nationality, ethnicity, sexual orientation or any other characteristic' (GBV SC Turkey, 2018: 24). Practitioners should ensure non-discrimination in all interactions with survivors and in all service provision. Hence they should (GBV SC Turkey, 2018: 24; GBV SC Iraq, n.d.: 14):

- Treat all survivors equally and in a dignified way.

- Maintain a non-judgmental manner.

- Not make assumptions about the history or background of a survivor.

- Be aware of their own prejudices and opinions about GBV, and not let these influence the way they treat a survivor.

- Not laugh or show any disrespect for the individual or his/her culture, family, or situation.

- Ensure they have been trained on human rights, humanitarian principles, and relevant agency non-discrimination policies.

\section{Confidentiality}

Confidentiality is an important part of the survivor-centred approach, and reflects the belief that people have the right to choose to whom they will, or will not, tell their story. 'Maintaining confidentiality means not disclosing any information at any time to any party without the informed consent of the person concerned. It promotes safety, trust and empowerment' (GBV Sub-Cluster Turkey Hub, 2018: 35). Zannoni (n.d.: 1) echoes this: 'Confidentiality plays a vital role in the recovery process because it helps establish an environment in which victims feel more comfortable seeking assistance, making connections, and exercising their power within their right to choose what information to share, with whom, when, and how'.

Confidentiality in the handling of GBV cases is both an ethical obligation and an operational necessity (Ribeiro \& Ponthoz, 2017). Ribeiro and Ponthoz (2017: 95) define confidentiality in the context of cases of sexual violence as follows:

Confidentiality is an ethical principle when documenting sexual violence that requires practitioners to protect information they gather on sexual violence, and apply that principle 
throughout the documentation exercise. Information about victims and other witnesses should be collected, used, shared and stored in a confidential manner. Depending on the context, confidentiality measures can be put in place to cover not only the identity of victims and other witnesses but also any information collected, referrals made and protective measures taken.

In practice this means, for example, that (Ribeiro \& Ponthoz, 2017: 95):

- those responsible for documentation are aware of what information is and is not to be taken, where and how it may be stored, and who has access to it;

- information protection measures are in place with regards to all identifying information on the victim/witness and their testimony, including the use of pseudonyms and coding systems for testimonial information as well as any referral options implemented or protective measures put in place;

- those responsible for documentation do not discuss case details with family, friends or colleagues who are not part of their team;

- they fully and clearly explain to survivors/witnesses the conditions and limits of confidentiality and ensure they give their informed consent to how their information is used.

These measures are further elaborated below under 'Data protection'. With regard to the final point above, there are certain circumstances in which confidentiality might need to be broken, such as (Ribeiro \& Ponthoz, 2017: 95):

- If the survivor is an adult who threatens their own life or who is directly threatening the safety of others, in which case referrals to lifesaving services can be sought.

- If the survivor is a child, when there are concerns for his/her health or safety.

- When there are mandatory reporting policies for cases of sexual exploitation and abuse that involve humanitarian workers.

A survivor should be informed about any exception to confidentiality. Documenters should be honest with them, and 'should never promise total anonymity or respect for confidentiality, as unintended breaches are always possible' (Ribeiro \& Ponthoz, 2017: 96). Where appropriate, this information should be provided in writing.

\section{Data protection}

Information provided by GBV survivors must be protected to prevent confidentiality breaches and protect survivors/witnesses from, for example, reprisal attacks by perpetrators, ostracism by the community. Threats to data can be both external (e.g. theft, destruction, hacking, modification) and internal (e.g. unauthorized access by internal individuals, accidental loss/destruction).

Measures for data protection need to be taken for both (Ribeiro \& Ponthoz, 2017: 111):

a) information 'in transit' - data that is being communicated (e.g. face to face, phone or video-call conversations, emails, chats, SMS, information being uploaded to or accessed from a cloud storage and their metadata)

b) information 'at rest' - data that is being stored (e.g. a hard-copies stored in a filing cabinet, digital files saved on a hard drive, cloud storage or digital devices and their metadata). 
Recommendations for practitioners to ensure information security include (GBV Sub-Cluster Turkey, 2018: 51; Ribeiro \& Ponthoz, 2017: 112):

- Staff charged with collecting the initial intake information from the survivor should be appropriately trained on how to fill out the forms and how to act in accordance with the guiding principles.

- The consent form, which normally includes names, should be stored separately from other documents. When possible, a coding system should be utilized for survivors' and staff's names. Each case file should be stored separately.

- Completed intake forms should never be transferred or shared between agencies in order to maintain the safety, security and confidentiality of information.

- Information should only be printed if absolutely necessary and all printed material should be destroyed when it is no longer needed.

- Information should not be emailed unless absolutely necessary. Any emails should never contain personal names nor contacts.

- Access to information should be controlled within every agency. This includes establishing clear responsibility for which staff can access or use survivor information, and limiting access to computers used to store confidential data.

- Information sharing should be on a strictly need-to-know basis, enabling the source of any information leak to be easily established

- Information stored electronically should be password protected.

- Clean desk policies should be adopted (i.e. no sensitive papers to be left on desk when offices are not in use).

\section{Referral}

This review focuses on documentation of GBV cases, but an essential activity following on from that is referral of survivors to appropriate support services, e.g. medical treatment, psychosocial counselling, safe shelters. The same principles need to be applied, in particular 'do no harm', as well as autonomy (respecting survivor's wishes) and informed consent. 'Providing information to survivors in an ethical, safe and confidential manner about their rights and options to access care is a cornerstone element of a survivor-centred referral system by which survivors can access the mix of services and support appropriate to their needs and wishes' (GBV SC Turkey, 2018: 34).

\section{GBV Information Management System (GBVIMS)}

\section{Background ${ }^{4}$}

The Gender-Based Violence Information Management System (GBVIMS) is a data management system that enables those providing services to GBV survivors to effectively and safely collect, store, analyse, and share data related to the reported incidents of GBV.

The GBVIMS emerged out of a consultation process carried out by WHO and UNFPA in 2005 and 2006, which called for a standardized system for GBV information management in

\footnotetext{
${ }^{4}$ This write-up on GBVIMS is largely adapted from: https://www.gbvims.com/what-is-gbvims/.
} 
humanitarian settings. Evaluation of GBV programmes had shown that different agencies and NGOs were using different terminology as well as classifying and counting procedures - making data compilation and analysis very difficult. The GBVIMS Global Team was put together in 2007, and began working on an information management system, which was piloted in Thailand, and subsequently modified and improved.

The GBVIMS provides a vehicle to ensure that service providers are respecting client confidentiality, obtaining client consent to share information, collecting data that is comparable across agencies and contexts, establishing protocols for sharing de-identified data, and obtaining information that can inform programming, resource allocation, and advocacy efforts.

The GBVIMS has already been implemented in agencies in Burundi, Chad, Central African Republic, Colombia, Côte d'Ivoire, Democratic Republic of Congo, Ethiopia, Guinea, Haiti, Iraq, Jordan, Kenya, Lebanon, Liberia, Mali, Nepal, Niger, Nigeria, Pakistan, Philippines, Sierra Leone, South Sudan, Tanzania, Thailand, Uganda, and Yemen, and continues to be rolled out more broadly.

\section{GBVIMS tools}

The GBVIMS is made up of four tools: the GBV Classification Tool, Intake and Consent Form, Incident Recorder, and the Information Sharing Protocol Template.

\section{GBV Classification Tool}

The GBV Classification Tool standardizes how GBV incident types are defined by using a set of six core types of GBV. ${ }^{5}$ It also standardizes the procedure by which incidents are classified through a process of elimination that determines the most specific incident type that applies to the reported incident.

The six core types of GBV included in the tool are: rape; sexual assault; physical assault; forced marriage; denial of resources, opportunities or services; and psychological/emotional abuse. Forms of GBV such as intimate partner violence and sexual slavery are not included as core types but can be analysed indirectly. ${ }^{6}$

\section{Intake and Consent Forms}

The Intake and Initial Assessment Form is a customizable template to be used by individuals interviewing GBV survivors reporting an incident. It is designed to collect a standard set of meaningful GBV indicators across all service providers while reducing variation in how data is collected. The Intake Form is comprised of six sections, each section containing fields collecting relevant and important information:

- Administrative Information

\footnotetext{
5 inspired by GBVIMS a localised version was developed for SINDH DAU and later the TASIR project of the British High Commission adapted it for Khyber Pakhtunkhwa and Punjab. It is not called GBVIMS as the GBV types go beyond what those in the GBVIMS: more types were added keeping in consideration the Pakistani context.

${ }^{6}$ For full details of the GBV Classification Tool see: http://gbvims.com/wp/wp-content/uploads/Annex-BClassification-Tool.pdf
} 
- Survivor Information

- Details of the Incident

- Alleged Perpetrator Information

- Planned Action/Action Taken

- Assessment Point

The Consent for Release of Information Form is a form requiring survivors to give their authorization for any of their information to be shared with other agencies or organizations. It is intended to ensure that the rights of the survivors to control their incident data are maintained and protected.

\section{Incident Recorder}

Once data has been collected using the Intake Form, service providers are required to input the data into the Incident Recorder (IR). The IR is an Excel spreadsheet that acts as a database for compiling and storing collected GBV data. It contains customizable dropdown lists to decrease input error and the time to input the data. The fields of the IR correspond directly with the fields of the Intake Form to make data input quick and easy. Based on the inputted data, the IR has builtin functions that will automatically generate GBV statistics, tables and charts that can be easily analysed, reported and shared.

\section{Information Sharing Protocol Template}

Without data protection and information-sharing protocols in place, organizations have little knowledge and trust of how others will use the information they share, leading many not to share GBV information. The Information Sharing Protocol Template has been designed to ensure that GBV data can be shared confidentially and compiled and analysed in a format that ensures survivor anonymity and the safety and security of all involved. The Protocol Template and accompanying guidance offer some ground rules and guiding principles on procedures for sharing non-identifiable data on reported cases of GBV. Ground rules include that no survivorspecific identifying information will be shared. ${ }^{7}$

\section{Primero/GBVIMS+}

Primero is an open-source software system designed to help GBV and Child Protection service providers securely and safely collect, store, manage, and share data on case management and incident monitoring. Developed in 2013, Primero houses different modules, one of which is GBVIMS+. GBVIMS + uses technological enhancements of the traditional (paper-based) GBVIMS to manage individual cases and services (case management) for survivors, as well as aggregate incident monitoring, while also facilitating internal compilation. The system also features heightened security, role-based access and viewing rights, and ways to accommodate different languages.

\footnotetext{
${ }^{7}$ For full details of Primero/GBVIMS+ see: http://www.gbvims.com/wp/wp-content/uploads/ISP-Package-August2014.pdf
} 


\section{Documentation with remote GBV services}

Remote GBV service delivery provides GBV services (predominately emotional support and case management) over a technology platform (i.e. hotline, chat, or SMS) rather than in person (IRC, 2018: 9). Two main methods by which GBV survivors can access support are through phonebased case management and by calling hotlines. The COVID-19 pandemic has led to both increased GBV (in particular domestic violence) and increased use of such remote service delivery, with often both survivor and case worker/responder based in their homes. ${ }^{8}$

Documentation and data storage in these situations poses additional challenges, which require appropriate measures to ensure confidentiality and data protection. A study of mobile and internet technology interventions to combat GBV in developing contexts, identified a number of potential ethical and security risks including (Hayes, n.d.: 31):

- threats to the privacy, confidentiality and security of online and mobile communications;

- the lack of clear standards surrounding the delivery of services using these technologies;

- risks and disruption to the organisation through attacks on the technology itself;

- the harassment and abuse of users.

Several of the organisations interviewed for the study highlighted the new ways in which online and mobile communications can potentially breach confidentiality, security and privacy through for instance, the interception of data, listening in to phone calls and accessing SMS messages (Hayes, n.d.: 31). Hayes (n.d.: 32) concluded the security and risk-management procedures of the organisations studied are not developing in line with the pace at which the technologies are evolving.

GBVIMS have produced guidance on remote GBV services focusing on phone-based case management and hotlines. ${ }^{9}$ The guidance includes information on how to:

- Establish and deliver phone-based GBV case management services

- Establish and deliver GBV hotline services

- Safety planning and other support for high-risk clients

- Update referral pathways

- Documentation and data storage

- Supervision and staff care for caseworkers and hotline staff

The guidance also looks at a variety of additional issues that may be encounter, including technology and network access, as well as safety planning.

A number of agencies including UNWomen and UNFPA have produced a decision tree on data collection on violence against women and COVID-19. It helps organizations with violence against

\footnotetext{
8 For more information about the impact of the COVID-19 pandemic see: Herbert, S. \& Marquette, H. (2021). COVID-19, governance, and conflict: emerging impacts and future evidence needs. K4D Emerging Issues Report 34. Brighton, UK: Institute of Development Studies. https://opendocs.ids.ac.uk/opendocs/handle/20.500.12413/16477
}

9 https://www.gbvims.com/covid-19-guidance-on-remote-gbv-services-focusing-on-phone-based-casemanagement-and-hotlines-out-now/ 
women programmes, national statistical offices, policymakers, and researchers decide when and how to best collect data on women's experiences of violence and their access and use of relevant services during the COVID-19 pandemic. The decision tree guides data collectors through the various considerations, viable options, and alternative data sources for obtaining information without jeopardizing participants' safety or the data's integrity. ${ }^{10}$

\section{Phone-based case management}

Phone-based case management is defined as follows (GBV AoR et al, 2021: 4):

(C)ase management that caseworkers provide over the phone to existing clients (or, in some cases when resources, safety and referral processes allow, new clients through direct referrals). It may be accessed through appointments agreed upon by the survivor and caseworker or through survivor-initiated calls when the caseworker is available (i.e., not open to the general public, or operating all hours).

Key considerations for those involved in phone-based GBV case management, particularly where caseworkers are also working remotely, include (GBV AoR et al, 2021: 57):

- Because the use of paper files introduces the opportunity for breach of confidentiality and data loss, it is strongly recommended that no hard copies of GBV case files or any other identifying information be stored in a caseworker's or supervisor's home. This includes consent, intake, and any other case management forms such as action plans and safety plans. Consent should instead be obtained verbally.

- Caseworkers who shift to remote case management should sign a data protection agreement.

- If survivors' phone numbers need to be recorded in order for caseworkers to remember how to contact their clients, it is recommended that caseworkers use anonymized codes in their phones. Separate password-protected files on caseworkers' laptops can connect names to phone numbers when absolutely needed.

- Information that must be recorded to facilitate a survivor referral or access to cash assistance (e.g. a survivors' name and phone number), should be written down and shared only to facilitate the assistance needed and then destroyed, preferably by burning so no evidence of documentation remains.

- Organizations typically reliant on paper files should consider shifting to new technologies that support safe and confidential collection of data during remote service delivery, such as PRIMERO/GBVIMS+. This has been designed to ensure that case management data cannot be obtained by a third party.

\section{Hotlines}

GBV hotlines are defined as follows (GBV AoR et al, 2021: 4):

an established phone service that provides crisis support and information to any survivor who calls. It is open to the general public and sometimes, but not always, for extended

\footnotetext{
10 https://www.unwomen.org/en/digital-library/publications/2020/07/decision-tree-data-collection-on-violenceagainst-women-and-covid-19
} 
hours. In many settings hotlines operate with toll-free numbers so that callers can avoid incurring fees.

Because hotlines use technology that can be monitored by perpetrators/abusers, particularly in intimate partner violence (IPV) situations, it is important to establish protocols to promote callers' safe use of the hotline. These protocols should be communicated to the caller from the outset (IRC, 2018: 63).

- No call back policy - Hotline staff should not call survivors back if there is an immediate risk to their safety, especially in situations of ongoing intimate partner violence. Ask survivors to call you back if you get disconnected.

- Remind survivors to delete the call record from the phone - One of the risks with hotlines is that perpetrators, particularly in situations of IPV, may monitor a survivor's phone use.

- Establish a code/red flag phrase - With survivors that receive ongoing support and case management via the hotline and have safety concerns at home, the survivor and caseworker should agree on a code that the survivor can use to signal to the hotline staff when the survivor thinks the call is being monitored and it is unsafe to talk. IPV perpetrators may not only monitor a survivor's phone use, but also the phone calls. If there are multiple hotline staff, the same code can be used across the programme (service) with different survivors to signal that they need to stop talking about violence and assume a different role/narrative, and then promptly end the call. For example, staff could tell all survivors to say, "You are the teacher from the school/clinic. I didn't sign up for the class," if she is in danger and needs to end the call.

- Any requested calls back from the programme should be accompanied by a safety plan.

Key considerations in relation to documentation and data storage for hotlines include (GBV AoR et al, 2021: 57-58):

- Most hotlines will want to keep a record of callers in order to monitor and analyse trends in hotline uptake. Even so, in hotline provision it is not good practice to record identifying information; the service operates as a crisis support whereby such information is not required in order to offer the service. Instead, hotline staff should note only anonymized call details, such as the date and time of the call; the length of the call; the types of incident being reported/reason for request for assistance; and any referrals offered. Hotline providers should not collect identifying information about callers, unless absolutely necessary in order to make a referral to services.

- For operators working from home, this information should be destroyed at the end of the call, or as soon as the referral is made. If this information cannot be safely destroyed, then it should be kept in a lockbox provided by the service agency until it can be destroyed.

- The only exception to this would be in contexts where hotlines are able to use the (paperbased) GBVIMS, or the Primero/GBVIMS+ (see above). It should be noted that for hotlines, however, operators will only need to fill out certain fields for the digital entry, and these need to be determined in advance and staff must receive sufficient training to adapt the digital tool for hotline use. 


\section{References}

CARE International (2014). Communications involving survivors of gender-based violence: Policy and guidelines. https://insights.careinternational.org.uk/images/in-practice/GBV/GBV-CommsPolicy-and-Guidelines.pdf

GBV AoR et al (2021). COVID-19 guidance on remote GBV services focusing on phone-based case management and hotlines. https:/www.gbvims.com/wp/wp-content/uploads/covidguidance-on-remote-gbv-services-04012021.pdf

GBV Sub-Cluster Iraq (n.d.). Standard Operating Procedures for prevention of and response to gender-based violence in Kurdistan Region of Iraq.

https://www.humanitarianresponse.info/sites/www.humanitarianresponse.info/files/documents/file s/gbv_sop_-_kri.pdf

GBV Sub-Cluster Turkey Hub-Syria (2018). Standard Operating Procedures for Gender-Based Violence Prevention and Response.

https://reliefweb.int/sites/reliefweb.int/files/resources/gbv_sc_sops_2018_english_final.pdf

Hayes, C. (n.d.) Tackling Gender-Based Violence with Technology: Case Studies of Mobile and Internet Technology Interventions in Developing Contexts. STATT

http://www.gendermatters.co.uk/pdfs/STATT\%20Tackling\%20GBV\%20with\%20Technology.pdf

IRC (2018). Guidelines for Mobile and Remote Gender-Based Violence (GBV) Service Delivery. International Rescue Committee. https://reliefweb.int/sites/reliefweb.int/files/resources/GBVMobile-and-Remote-Service-Delivery-Guidelines_ffinal.pdf

Ribeiro, S. \& Ponthoz, D. (2017). International Protocol on the Documentation and Investigation of Sexual Violence in Conflict. Best Practice on the Documentation of Sexual Violence as a Crime or Violation of International Law. Second Edition, UK Foreign \& Commonwealth Office. https://assets.publishing.service.gov.uk/government/uploads/system/uploads/attachment_data/fil e/598335/International_Protocol_2017_2nd_Edition.pdf

SJAC (n.d.). Documenting sexual and gender based violence. Syria Justice and Accountability Centre. https://www.irex.org/sites/default/files/node/resource/sjac-gender-sgbv-documentationpolicy-executive-summary.pdf

UN OHCHR (2019). Protection of victims of sexual violence: Lessons learned. Office of the United Nations High Commissioner for Human Rights.

https://www.ohchr.org/Documents/lssues/Women/WRGS/ReportLessonsLearned.pdf

WHO \& UNODC (2014). Strengthening the Medico-Legal Response to Sexual Violence. World Health Organization (WHO) and UN Office on Drugs and Crime (UNODC).

https://www.unodc.org/documents/publications/UNODC_WHO_Sexual_Violence_toolkit_eng.pdf

Zannoni, J. (n.d.). Strengthening Sexual Assault Victims' Right to Privacy. Office for Victims of Crime (OVC), USA. https://www.ojp.gov/pdffiles1/Digitization/226501NCJRS.pdf 


\section{Key websites}

- Gender-Based Violence Information Management System:

https://www.gbvims.com/what-is-gbvims/

\section{Suggested citation}

Idris, I. (2021). Documentation of survivors of gender-based violence (GBV). K4D Helpdesk Report. Brighton, UK: Institute of Development Studies. DOI: 10.19088/K4D.2021.103

\section{About this report}

This report is based on six days of desk-based research. The K4D research helpdesk provides rapid syntheses of a selection of recent relevant literature and international expert thinking in response to specific questions relating to international development. For any enquiries, contact helpdesk@k4d.info.

K4D services are provided by a consortium of leading organisations working in international development, led by the Institute of Development Studies (IDS), with Education Development Trust, Itad, University of Leeds Nuffield Centre for International Health and Development, Liverpool School of Tropical Medicine (LSTM), University of Birmingham International Development Department (IDD) and the University of Manchester Humanitarian and Conflict Response Institute (HCRI).

This report was prepared for the UK Government's Foreign, Commonwealth and Development Office (FCDO) and its partners in support of pro-poor programmes. Except where otherwise stated, it is licensed for non-commercial purposes under the terms of the Open Government Licence v3.0. K4D cannot be held responsible for errors, omissions or any consequences arising from the use of information contained in this report. Any views and opinions expressed do not necessarily reflect those of FCDO, K4D or any other contributing organisation.

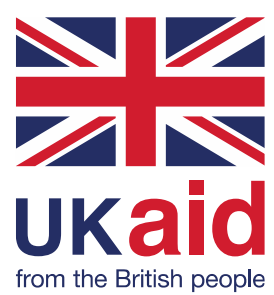

(C) Crown copyright 2021. 